\title{
Counting steps in research: A comparison of accelerometry and pedometry
}

\author{
Melody Oliver ${ }^{1,2}$, Hannah M Badland ${ }^{1,3}$, Janine Shepherd ${ }^{1}$, Grant M Schofield ${ }^{1}$ \\ ${ }^{1}$ Centre for Physical Activity and Nutrition, Auckland University of Technology, Auckland, New Zealand \\ ${ }^{2}$ School of Public Health and Psychosocial Studies, Auckland University of Technology, Auckland, New Zealand \\ ${ }^{3}$ Division of Population Health, University College London, London, United Kingdom \\ Email: melody.oliver@aut.ac.nz
}

Received 3 March 2011; revised 10 May 2011; accepted 12 May 2011.

\begin{abstract}
The objective of this study was to assess the validity of the step count functions in Actical accelerometers and activPAL inclinometers, compared with pedometer-derived step count data. Firstly, directly observed step counts over 3 treadmill speeds were compared with steps collected from 3 pedometers, accelerometers, and inclinometers in 10 adults. Secondly, step count data were derived from 22 participants who wore a pedometer, accelerometer, and inclinometer over 48 hours. Agreement between measurement tools was determined. All monitors appropriately measured steps in the laboratory conditions. In free living conditions, the mean percentage differences with pedometer-determined step counts were $-7.3 \%$ and $7.0 \%$ for the Actical and activPAL monitors, respectively. With the exception of slow walking for the Actical units $($ ICC $<\mathbf{0 . 0 0 1}$ ), acceptable reliability was found within units for all treadmill speeds, and across units during the free living condition. The 95\% prediction interval ranges were wide, ranging from $-\mathbf{6 8 . 8} \%$ to $\mathbf{5 4 . 2 \%}$ for the Acticals, and from $-39.1 \%$ to $53.2 \%$ for the activPALs. Step counts gathered from Actical and activPAL units should not be used interchangeably with pedometer-derived step count data.
\end{abstract}

Keywords: Physical Activity; Measurement; Pedometer; Inclinometer; Accelerometer; Validation; Reliability

\section{INTRODUCTION}

Accurate quantification of free-living physical activity is important to enable researchers to derive reliable information on associations with health outcomes and develop public health recommendations, identify determinants of activity and individuals/population groups at risk of insufficient activity for health, and to accurately assess intervention effectiveness. Accelerometers are increasingly being used for this purpose, due to the utility of these monitors to provide objective and precise information on activity intensity accumulated over sustained periods. No best practice has yet been determined for accelerometer data treatment; inconsistencies in approaches to data reduction have made it especially challenging to accurately track activity behaviors or compare results across studies. Indeed, increasingly sensitive measurement instruments may offer a decrease in specificity of measurement especially at the lower intensity end of measurement. Moreover, understanding and interpreting physical activity intensity concepts can be challenging, making it difficult to translate and disseminate practical health promotion messages. In contrast, the measurement of physical activity in the form of steps is relatively straightforward to measure via pedometry, and the data are (relatively) simple to analyze and disseminate and make inferences to the general public. Body composition-referenced step count recommendations have been developed [1] and pedometer-based programs have been shown to be effective in increasing physical activity (especially where step goals are included) and improving health outcomes (e.g., reducing body mass index and blood pressure) [2]

Accordingly there is opportunity to augment more complex physical activity measures (accelerometry, inclinometry) with step counts. Two commercially available monitors exist that provide both step count and more complex movement data: 1) Actical accelerometers now offer the ability to assess steps accumulated in addition to existing accelerometry-based physical activity assessment, and 2) the activPAL is also an accelerometer-based monitor that includes an inclinometer; for ease of differentiation between units these will be termed inclinometers hereafter. These inclinometers provide 
date and time stamped information on time spent sitting, lying, standing, walking, and number of steps accumulated. The Actical accelerometer has been shown to be valid for estimating physical activity intensity in adults [3]. Validity of the new step-count function in Actical accelerometers has been assessed using laboratory-based activities [4], however to our knowledge, no assessment of the validity of this function in measuring steps accumulated during free-living physical activity over sustained periods of time (i.e., $>1 \mathrm{~d}$ ) has been conducted. activPAL monitors have shown acceptable reliability [5,6] and validity [7] for measuring time spent being sedentary and active (including walking) under controlled/laboratory conditions. Evidence suggests that the accuracy of these monitors may be lowest when assessing time spent walking [5]. The only studies to assess the activPAL step-count function in measuring actual steps taken have also been undertaken in laboratory/controlled conditions [8,9]. Findings indicate these monitors are reliable [8] and accurate to within $1.3 \%$ for treadmill and outdoor walking $[8,9]$.

Generally, laboratory-based testing has provided sufficient evidence for the validity of both the Actical and activPAL monitors to accurately assess steps in adults. This research now needs to be extended to application in free-living situations for durations greater than the 6 hours employed to date. Research aims were thus to assess the reliability, validity, and agreement of step-count data gathered from Actical accelerometers and activPAL inclinometers with standard practice for step count measurement (pedometry) in free-living conditions over multiple days.

\section{METHODS}

\subsection{Participants}

Convenience samples of university employees in Auckland, New Zealand were recruited for each testing stage. For the laboratory-based testing, 10 university staff were invited to participate. Participants for the free-living testing were recruited as part of a wider study investigating measurement of sedentary behavior [10,11]. As such, these participants were included only if they reported spending the majority of their occupational time sitting. No other inclusion/exclusion criteria were employed. Ethical approval to conduct the study was provided by Auckland University of Technology Ethics Committee (AUTEC reference 09/253, granted 25 November 2009). Written informed consent was gathered from all participants. Data were collected on weekdays between December 2009 and March 2010.

\subsection{Measurement Tools}

Three Actical accelerometers (Mini-Mitter, Respironics
Inc Company, Bend, OR), three activPAL inclinometers (PAL Technologies Ltd, Glasgow), and three Yamax Digiwalker CW-700 pedometers (Yamax Corp., Kumamoto, Japan) were used in the current study. Yamax Digiwalker (e.g., SW-701, SW-200) pedometers are valid and reliable for measuring steps in adults $[12,13]$. The CW-700 improves over previous Digiwalker models by including a multi-day-memory function, but otherwise the mechanical properties of these units are identical to earlier models. Performance of all units was first assessed in laboratory conditions prior to application in free-living conditions.

\subsection{Procedures}

\subsubsection{Laboratory Testing}

Tests were conducted on a Powerjog GX C200 motorized treadmill (PowerSport International Inc., Bridgend, UK). Participants underwent a $5 \mathrm{~min}$ familiarization procedure, including practicing starting and stopping at each speed. Units were then attached, with the three activPAL units placed medially and vertically on the right vastus medialis with Physiomed TheraFIX Underwrap tape, and the three pedometers and accelerometers attached to elastic belts and placed above the right iliac crest. Participants then walked for $5 \mathrm{~min}$ at three speeds: $54 \mathrm{~m} \cdot \mathrm{min}^{-1}, 107 \mathrm{~m} \cdot \mathrm{min}^{-1}$, and $80 \mathrm{~m} \cdot \mathrm{min}^{-1}$, with a $1 \mathrm{~min}$ rest between each test. Walking speeds were identified as being the lowest, highest, and median values used in previous research $[8,12]$. Pedometers were set to 0 before each trial and readings from each pedometer were taken immediately post each test. During the tests, one research assistant counted down start and stop times, recorded measurement times, and pedometer steps, and a second research assistant counted observed steps using a manual step counter.

\subsubsection{Free-Living Testing}

Participants were provided with one activPAL inclinometer and tape, one Actical accelerometer, one Yamax pedometer, and unit wearing instructions by a trained research assistant. On delivery, pedometers were set to zero, sealed with tamper-evident tape, then placed on an elastic belt at the right hip above the iliac crest and secured with an additional safety strap. Accelerometers were attached to the elastic belts in line with the pedometer. The inclinometer was attached medially on the right vastus medialis, secured in place with Physiomed TheraFIX Underwrap tape. Participants were asked to wear all units for the following 48 hours and to remove them only when bathing or sleeping. Participants also completed a paper-based compliance diary for the $48 \mathrm{~h}$ period they wore the measurement units (including researcher-reported delivery and collection times and pedometer steps accumulated at the time of pedometer 
collection), and were called once during the measurement period to confirm monitor wear. Monitors were collected by the research assistant exactly $48 \mathrm{~h}$ after they were delivered. On collection, the researcher recorded the time of unit removal and pedometer steps accumulated for that day. Participants reported their height in meters and weight in kilograms. Body mass index (BMI) was calculated as $\mathrm{kg} \cdot \mathrm{m}^{-2}$, and thresholds of $25 \mathrm{~kg} \cdot \mathrm{m}^{-2}$ and $30 \mathrm{~kg} \cdot \mathrm{m}^{-2}$ were used to define overweight and obesity, respectively.

\subsection{Statistical Analyses}

\subsubsection{Laboratory Testing}

Accelerometer and inclinometer data were downloaded using Actical 2.04 and activPAL 5.8.3.5, respectively, and combined by time in Microsoft Excel. Step count data for the accelerometers and inclinometers were then summed for each $5 \mathrm{~min}$ bout and matched with the pedometer and observed step counts. Intraclass correlation coefficients (ICC) for step counts from each unit at each speed were calculated. Agreement between observed steps (criterion) and step counts from pedometers, accelerometers, and inclinometers (comparison measures) was assessed using the Bland-Altman method $[14,15]$. Initially, Bland-Altman plots and associated lowess curves were produced and assessed for each of the measurement units against the observed steps at each ambulatory speed to determine whether combined or subgrouped analyses were necessary. Average values of the observed step counts and each comparison measure were compared against the difference of the observed step counts and steps from each comparison measure. Percentage differences between the observed steps and comparison measures were then calculated and the corresponding mean differences and $95 \%$ limits of agreement $( \pm 1.96 \mathrm{SE})$ calculated and plotted. Equality in variance between percentage differences in step count readings at each gait speed was formally determined for each comparison measure using the Brown and Forsythe test for equality [16]. Statistical analyses were undertaken using Stata IC version 10 (StataCorp, TX) and $\alpha=$ 0.05 was used to determine statistical significance.

\subsubsection{Free-living Testing}

Self-reported compliance in wearing the units was compared with information gathered during the random phone calls for accuracy. Participants not meeting minimal compliance criteria of wearing the units for at least one full ( $\geq 10 \mathrm{~h}$ on day 2) and one partial (afternoon of day 1 or morning of day 3 ) day were excluded from further analyses. Accelerometer and inclinometer data were downloaded using the Actical and activPAL software, respectively, and then combined by date and time in Excel. Data for the $48 \mathrm{~h}$ measurement period were manu- ally extracted for each individual using the unit delivery and collection times from the compliance diary, and step counts for the measurement period were summed from the extracted data. Researcher-reported pedometer step counts for the day of collection (day 3) were obtained from the compliance diary, and steps for previous days (days 1 and 2) were gathered from the pedometer memory. Days where pedometer step counts were greater than 30000 or less than 1000 (or proportionate values for partial days) were considered outliers and removed from analyses [17]. Only participants with at least one full and one partial day of pedometer data remaining and concurrent accelerometer and/or inclinometer data were included in further analyses.

Remaining step count data were combined for each measurement tool. Average pedometer steps $\cdot h^{-1}$ were calculated and differences by sex, BMI status (dichotomized as normal, overweight/obese), and age group (dichotomized as 20-43y, 44-58y) were determined using independent $t$ tests. Pedometer data were matched with available accelerometer and inclinometer data by times worn. Associations between the unit types for steps counted (i.e., pedometer, Actical accelerometer, activPAL inclinometer) was determined by ICC. Agreement between step counts from the pedometer and 1) Actical accelerometer step counts and 2) activPAL inclinometer step counts were assessed using the BlandAltman method [14,15] as per the laboratory testing analyses above.

\section{RESULTS}

\subsection{Laboratory Testing}

Ten adults (9 females) completed the measurement protocol. Table 1 shows the mean (SD) of the observed step counts and step counts from comparison measures at each gait speed, the mean (SD) for the percentage differences between observed steps and comparison measures, and the related ICC values for steps counted. With the exception of the accelerometers at slow walking speeds (ICC < 0.001), units demonstrated acceptable reliability across all walking speeds (ICC $=0.335-0.998$ ). Unit reliability increased with walking speed for pedometers and accelerometers, while activPAL performance was consistent across all pace conditions (ICC $=$ 0.998). Visual analysis of Bland-Altman plots and associated lowess curves indicated that the use of combined analyses was appropriate. activPAL units performed to $98.2 \%$ accuracy across all pace conditions. Significant heterogeneity was found in the variances of observedcomparison measurements over gait speed for the pedometers and accelerometers (Brown-Forsythe test $\mathrm{p}<$ 0.001 ) indicating that the accuracy of these measurement tools was dependent on gait speed. Figure 1 shows the 
Table 1. Median (SD) of steps observed and recorded from the Yamax Digiwalker pedometer, Actical accelerometer, and activPAL inclinometer under three treadmill pace conditions, and relative percentage differences between observed steps and comparison units in 10 adults.

\begin{tabular}{|c|c|c|c|c|c|c|c|c|c|c|c|c|c|c|c|c|c|}
\hline \multirow{3}{*}{$\begin{array}{l}\begin{array}{c}\text { Treadmill } \\
\text { speed }^{\mathrm{a}}\end{array} \\
\text { Slow }\end{array}$} & \multirow{2}{*}{\multicolumn{2}{|c|}{$\begin{array}{l}\text { OB } \\
\text { Steps } \\
\text { ean (SD) }\end{array}$}} & \multicolumn{5}{|c|}{ Yamax Pedometer } & \multicolumn{5}{|c|}{ Actical accelerometer } & \multicolumn{5}{|c|}{ activPAL inclinometer } \\
\hline & & & \multicolumn{2}{|c|}{$\begin{array}{c}\text { Steps } \\
\text { mean (SD) }\end{array}$} & \multicolumn{2}{|c|}{$\begin{array}{c}\% \text { difference } \\
\text { from OB } \\
\text { mean }(\mathrm{SD})\end{array}$} & \multirow{2}{*}{$\begin{array}{r}\text { ICC } \\
0.387\end{array}$} & \multicolumn{2}{|c|}{$\begin{array}{c}\text { Steps } \\
\text { mean (SD) }\end{array}$} & \multicolumn{2}{|c|}{$\begin{array}{l}\% \text { difference } \\
\text { from OB } \\
\text { mean (SD) }\end{array}$} & \multirow{2}{*}{$\begin{array}{c}\text { ICC } \\
<0.001\end{array}$} & \multicolumn{2}{|c|}{$\begin{array}{c}\text { Steps } \\
\text { mean (SD) }\end{array}$} & \multicolumn{2}{|c|}{$\begin{array}{c}\% \text { difference } \\
\text { from OB } \\
\text { mean }(\mathrm{SD})\end{array}$} & \multirow{2}{*}{$\begin{array}{r}\text { ICC } \\
0.998\end{array}$} \\
\hline & 477 & $(21.6)$ & 394 & (109.4) & -17.4 & $(23.1)$ & & 394 & $(93.8)$ & -17.2 & $(19.6)$ & & 468 & (21.6) & -1.9 & $(0.3)$ & \\
\hline Medium & 568 & $(29.5)$ & 557 & $(80.7)$ & -2.1 & $(12.7)$ & 0.369 & 546 & $(63.1)$ & -3.8 & $(9.3)$ & 0.335 & 557 & $(28.8)$ & -1.9 & $(0.4)$ & 0.998 \\
\hline Fast & 627 & $(32.1)$ & 631 & $(33.1)$ & 0.5 & $(0.4)$ & 0.997 & 621 & $(38.2)$ & -1.0 & (3.8) & 0.590 & 616 & (31.5) & -1.8 & $(0.4)$ & 0.998 \\
\hline Combined & 557 & (68.3) & 527 & (127.6) & -6.3 & $(17.0)$ & $\mathrm{n} / \mathrm{a}$ & 521 & (116.8) & -7.3 & (14.4) & $\mathrm{n} / \mathrm{a}$ & 547 & (67.1) & -1.8 & $(0.4)$ & $\mathrm{n} / \mathrm{a}$ \\
\hline
\end{tabular}

Notes: $\mathrm{ICC}=$ Intraclass Correlation Coefficient, $\mathrm{n} / \mathrm{a}=$ not applicable, $\mathrm{OB}=$ Observed Steps, $\mathrm{SD}=\mathrm{Standard}$ Deviation; $\mathrm{a} . \mathrm{Slow}=54 \mathrm{~m} \cdot \mathrm{min}^{-1}, \mathrm{Medium}=80$ $\mathrm{m} \cdot \mathrm{min}^{-1}$, Fast $=107 \mathrm{~m} \cdot \mathrm{min}$

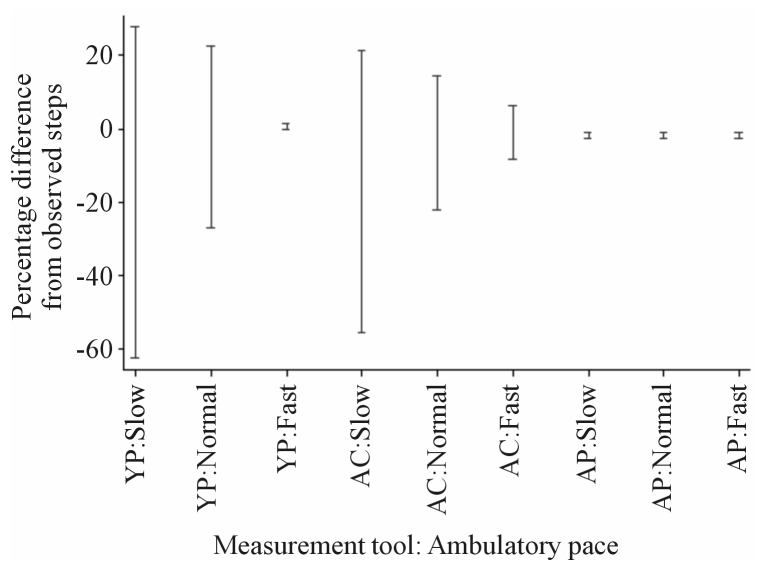

Figure 1. 95\% limits of agreement for difference between observed step counts and Yamax Pedometer step counts, Actical accelerometer step counts, and activPAL inclinometer step counts over 5 minutes treadmill walking, by ambulatory gait speed.

95\% limits of agreement for differences between the observed step counts and each of the measurement tools, by ambulatory gait speed. Performance of all units was similar to previous validation studies $[4,8,9,12]$ and so these were considered acceptable for further testing in free-living conditions.

\subsection{Free-Living Testing}

Twenty-five adults aged $41.6 \pm 11.8 \mathrm{y}$ participated in the study, with an average BMI of $26.1 \pm 5.0 \mathrm{~kg} \cdot \mathrm{m}^{-2}$. Selfreported compliance matched that reported in the phone calls, and participants wore the units for an average of $26.8 \mathrm{~h}$ (range $15.7-34.0 \mathrm{~h}$ ) over the $48 \mathrm{~h}$ period. In total, 22 participants met the minimum pedometer compliance criteria. Of these, corresponding accelerometer and inclinometer data were gathered from 22 and 19 participants, respectively. Table 2 displays the participant characteristics and average step counts for participants meeting the pedometer data inclusion criteria. Average (minimum, maximum) wear times for pedometer and accelerometer data included in analyses was $24.5 \mathrm{~h}(15.7$ $\mathrm{h}, 31.3 \mathrm{~h})$, and for inclinometer data was $22.3 \mathrm{~h}(13.3 \mathrm{~h}$, $31.2 \mathrm{~h}$ ). No significant differences in average pedometer steps $\cdot \mathrm{h}^{-1}$ were found for sex, age, or BMI status ( $\mathrm{p}>$ $0.05)$. A high degree of correlation was found across all units for the within-participant step counts (ICC $=0.850$, 0.807 , and 0.666 for pedometer-Actical, pedometeractivPAL, and Actical-activPAL, respectively).

Figure 2 shows the Bland Altman plot for agreement and associated 95\% limits of agreement for the Actical and activPAL step counts relative to the pedometer- determined step counts. The mean percentage differences with pedometer-determined step counts were $-7.3 \%$ and $7.0 \%$ for the Actical and activPAL monitors, respectively. The $95 \%$ prediction interval ranges were wide for both

Table 2. Descriptive characteristics and step counts for free-living study participants meeting pedometer data inclusion criteria.

\begin{tabular}{|c|c|c|}
\hline Variable & $\mathrm{N}$ & $(\%)$ \\
\hline \multicolumn{3}{|l|}{ Sex } \\
\hline Male & 6 & $(27.3)$ \\
\hline Female & 16 & $(72.7)$ \\
\hline \multicolumn{3}{|l|}{ Age (yr) } \\
\hline $20-43$ & 13 & $(59.1)$ \\
\hline $44-58$ & 9 & $(40.9)$ \\
\hline \multicolumn{3}{|l|}{ BMI classification } \\
\hline $\begin{array}{l}\text { Normal/underweight } \\
\left(<25 \mathrm{~kg} \cdot \mathrm{m}^{-2}\right)\end{array}$ & 12 & $(54.5)$ \\
\hline Overweight $\left(25-29 \mathrm{~kg} \cdot \mathrm{m}^{-2}\right)$ & 5 & $(22.7)$ \\
\hline Obese $\left(\geq 30 \mathrm{~kg} \cdot \mathrm{m}^{-2}\right)$ & 5 & $(22.7)$ \\
\hline Step counts & Mean & $\begin{array}{l}\text { (minimum, } \\
\text { maximum) }\end{array}$ \\
\hline \multicolumn{3}{|l|}{ Total step counts } \\
\hline Pedometer $^{\mathrm{a}}$ & 14207 & $(6335,36290)$ \\
\hline Accelerometer & 13118 & $(3859,34113)$ \\
\hline Pedometer $^{b}$ & 12814 & $(7846,36290)$ \\
\hline Inclinometer & 13272 & $(6820,33232)$ \\
\hline \multicolumn{3}{|l|}{ Average step counts (steps $\cdot h^{-1}$ ) } \\
\hline Pedometer $^{\mathrm{a}}$ & 586 & $(277,1788)$ \\
\hline Accelerometer & 544 & $(153,1680)$ \\
\hline Pedometer $^{b}$ & 600 & $(277,1831)$ \\
\hline Inclinometer & 617 & $(303,1677)$ \\
\hline
\end{tabular}

${ }^{a}$ Matched for times worn with Actical accelerometer; ${ }^{b}$ Matched for times worn with activPAL inclinometer 
comparison measures, however, ranging from $-68.8 \%$ to $54.2 \%$ for the Actical accelerometer (width $122.9 \%$ ), and from $-39.1 \%$ to $53.2 \%$ for the activPAL inclinometer (width 92.3\%). Within-individual inconsistencies in the direction and scale of misclassification for the comparison units were also found; Figure 3 shows the percentage difference between the comparison measures and the average steps gathered from the pedometer and each comparison measure for each participant.

\section{DISCUSSION}

To our knowledge, this is the first study to investigate the agreement between pedometer steps and steps gathered from Actical accelerometers and activPAL inclinometers in free-living conditions over multiple days. Our initial laboratory testing showed similar results to previous research, with step count underestimation in Yamax pedometers $[8,9,12]$ and Actical accelerometers [4] at slower speeds but acceptable accuracy and reliability for medium speed and fast walking. For the activPAL steps we found a high degree of accuracy and reliability $(>98 \%$, ICC $=0.998)$ with observed steps for all walking speeds [8,9]. In line with Esliger et al. [4] we also noted considerable variability in within-individual inconsistencies in the percentage differences for the Actical and activPAL monitors, indicating there was no consistent pattern that could be related to the unit or individual participants.

Considering the laboratory-based results of the current research and previous studies [8,9], it is likely that the activPAL yielded the most accurate measure of steps accumulated in free living conditions, which are likely to include walking at lower speeds. The only other study to consider the accuracy of activPAL units in free living conditions (albeit for approximately $6 \mathrm{~h}$ only) showed a high degree of accuracy in classifying time spent stepping [7]. A second-by second analysis of activPAL- derived classification of time spent stepping, sitting/lying, and standing showed an overall agreement of $95 \%$ with directly observed activities, however the limits of agreement were widest for walking (range $-16.1 \%$ to $12.1 \%$ ) [5].

While findings from the current study corroborate previous research findings, the reduced accuracy of the Yamax Digiwalker pedometers at lower speeds clearly limits their accuracy in measuring actual steps taken in freeliving conditions, and therefore their utility as a criterion/comparison measure in the current study. Pedometer steps are the most common and widely referenced basic unit of objectively measured physical activity. As such, the inclusion of pedometers in the current study was still deemed important, to determine whether steps gathered from Actical and activPAL units could appropriately be used interchangeably with pedometer steps as a measure

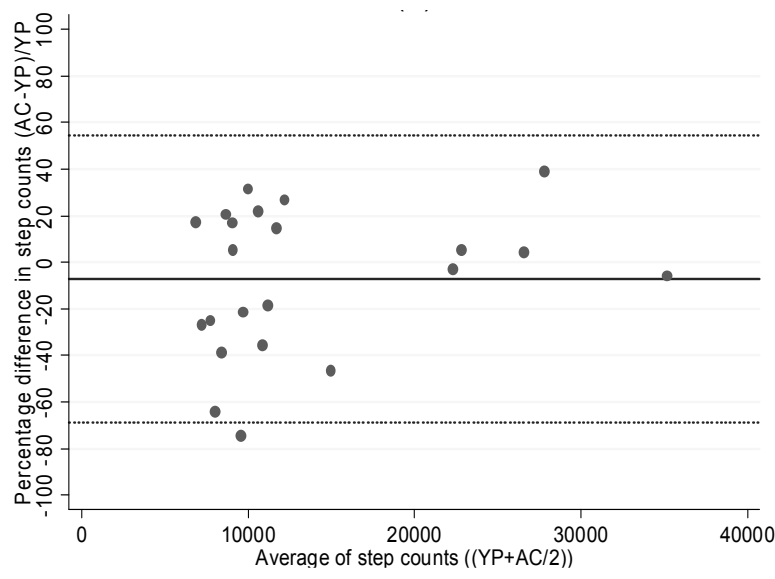

(a)

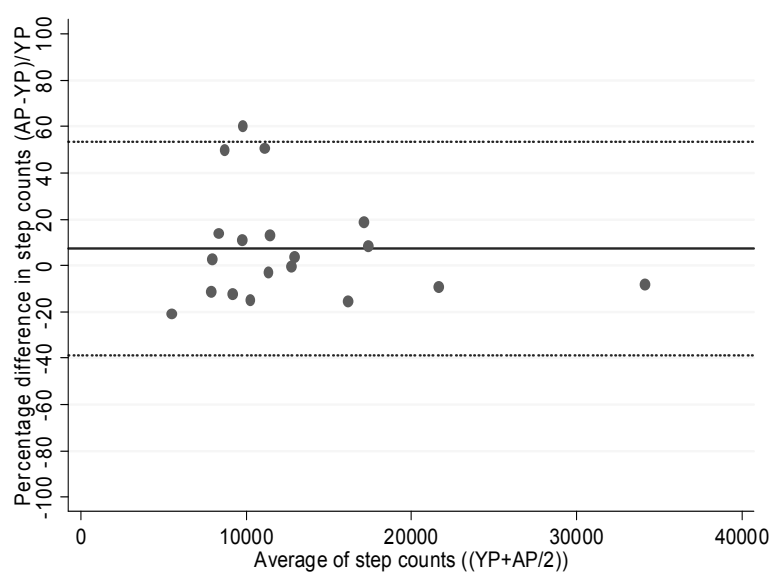

(b)

Figure 2. Bland Altman plots demonstrating agreement between Yamax Digiwalker pedometer steps and Actical accelerometer steps (a), and activPAL inclinometer steps (b).

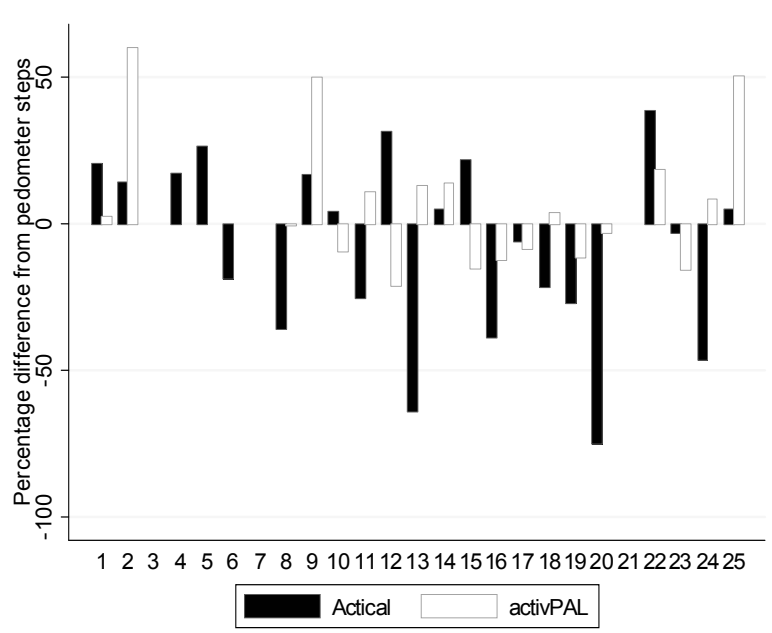

Figure 3. Percentage difference between Yamax Digiwalker pedometer steps and comparison measures (Actical accelerometer, activPAL inclinometer) for each participant (ID 1.25) during the free living condition. 
of health-related physical activity.

The limits of agreement with pedometer steps for both the Actical accelerometers and activPAL inclinometers were wide, at $123 \%$ and $92 \%$, respectively. As we may expect daily step counts of between 7000 and 13000 in healthy adults [18], an example of the outcome of this is provided using the mean of these values: Findings from the current study can be interpreted as meaning that when an observed step count of 10000 is recorded by a pedometer, in $95 \%$ of instances the Actical monitors will record a step count between 3120 and 15420 steps (width 12300 steps), and the activPAL between 6090 and 15320 steps (width 9230 steps). Detecting changes in daily step counts of 2500 steps as a result of an intervention has been associated with improvements in health outcomes [19] and generally equates to one 'band' of physical activity level classification [20]. Such changes in daily step counts may be considered clinically important. The results from the present study clearly show that the prediction widths observed for the Actical and activPAL units were too great for these units to be considered as being in agreement with pedometer steps measured. Based on our findings we conclude that step count data observed in Actical accelerometers and activPAL inclinometers cannot be used interchangeably with pedometer steps, nor should they be used to classify individual activity levels based on step count criteria.

Taken together, the results of the current study indicate that the activPAL monitor will provide the best representation of actual steps taken in free-living situations. This is not surprising given the placement of the unit on a participant's upper leg, where the detection of walking at any velocity is based on large gross limb movement. The hip-mounted pedometer and accelerometer must rely on more subtle vertical displacements to detect a step, and these displacements reduce when walking velocity decreases. The Digiwalker is a spring-levered pedometer whereby upon vertical displacement, an internal spring-suspended arm moves vertically, opening and closing an electrical circuit to register a step. There is some evidence that piezoelectric pedometers (comprising a piezoelectric crystal which is moved in response to acceleration, e.g., New Lifestyles, Kenz Lifecorder) may be more accurate at slower speeds than other pedometer technologies, especially in overweight or obese individuals $[12,21]$. Accurate quantification of low velocity physical activity may be a very important part of understanding the differences between lean and obese adults [22]. Levine et al. [22] observed that lean subjects accumulated an extra 3.5 miles $\cdot \mathrm{d}^{-1}$ of low velocity walking $\left(\sim 1\right.$ mile $\left.\cdot \mathrm{h}^{-1}\right)$ than obese subjects. It is also plausible that slower velocity walking provides a lower metabolic benefit than walking at higher speeds and the under- reading of pedometer steps at lower velocities provides a reasonable proxy for such a difference in benefit.

Additionally, activPAL monitors are not necessarily suitable for all types of physical activity measurement in the same way pedometers, and to a certain extent accelerometers, may be. While they may be most accurate in step count assessment, they are not able to assess subtle movements such as shifting weight while standing, and measurement is limited to the site/limb of placement (e.g., right leg movement only). Notably, these limitations also apply to pedometers and accelerometers (albeit some accelerometers are sensitive enough to capture small movements such as weight shifts). It is also worthwhile noting that this lack of sensitivity actually improves accuracy in step counting; for example, Maddocks et al. [9] found that vibrations caused by car travel influenced activity data collected by Digiwalker pedometers and PALlite accelerometers, while no activity was recorded by activPAL units under the same conditions. In comparison to pedometers, activPAL monitors are expensive (approximately \$US400 per unit) and mounting the unit with tape on a participant's thigh is difficult and more invasive than fitting belt-mounted pedometers or accelerometers. When activity measurement is over multiple days, monitor removal and reattachment needs to be managed by the participants themselves, and this may present both compliance and other measurement consistency problems. These issues make larger population epidemiology, and work with younger and older populations less feasible. Finally, it is worth noting that the relatively small sample in this study was predominantly female, employed, and likely to be well educated, thus limiting the generalisability of the results from the free-living conditions. Further research would benefit from including a larger and more representative sample in order to capture a broad variety of daily activities across a wide range of population groups.

\section{CONCLUSION}

The three types of units accurately measured step counts under laboratory conditions, but when the step counts derived from Actical accelerometers and activPAL inclinometers were compared with the pedometer values under free living conditions, considerable disagreement was evident. Therefore, these different devices are not interchangeable when measuring step counts, and Actical and activPAL units should not be used to classify physical activity levels based on existing step count thresholds. Pedometers remain the most cost effective and simple method of step count measurement within free living settings, while activPAL inclinometers are the most accurate for assessing steps gathered over a range 
of walking speeds.

\section{ACKNOWLEDGEMENTS}

At the time of writing, Melody Oliver and Hannah Badland were supported by National Heart Foundation of New Zealand research fellowships. A Faculty of Health and Environmental Sciences Auckland University of Technology Summer Studentship supported Janine Shepherd.

\section{REFERENCES}

[1] Tudor-Locke, C., Ainsworth, B.E., Whitt, M.C., Thompson, R.W., Addy, C.L. and Jones, D.A. (2001) The relationship between pedometer-determined ambulatory activity and body composition variables. International Journal of Obesity, 25, 1571-1578.

doi:10.1038/sj.ijo.0801783

[2] Bravata, D.M., Smith-Spangler, C., Sundaram, V., Gienger, A.L., Lin, N., Lewis, R., et al. (2007) Using pedometers to increase physical activity and improve health. A systematic review. Journal of the American Medical Association, 298, 2296-2304. doi:10.1001/jama.298.19.2296

[3] Heil, D.P. (2006) Predicting activity energy expenditure using the Actical ${ }^{\circledR}$ activity monitor. Research Quarterly for Exercise and Sport, 77, 64-80.

[4] Esliger, D.W., Probert, A., Gorber, S.C., Bryan, S., Laviolette, M. and Tremblay, M.S. (2007) Validity of the Actical accelerometer step-count function. Medicine \& Science in Sports \& Exercise, 39, 1200-1204. doi:10.1249/mss.0b013e3804ec4e9

[5] Grant, P.M., Ryan, C.G., Tigbe, W.W. and Granat, M.H. (2006) The validation of a novel activity monitor in the measurement of posture and motion during everyday activities British Journal of Sports Medicine, 40, 992997. doi:10.1136/bjsm.2006.030262

[6] Busse, M.E., van Deursen, R.W. and Wiles, C.M. (2009) Real- life step and activity measurement: Reliability and validity. Journal of Medical Engineering \& Technology, 33, 33-41. doi:10.1080/03091900701682606

[7] Godfrey, A., Culhane, K.M. and Lyons, G.M. (2007) Comparison of the performance of the activPAL ${ }^{\mathrm{TM}}$ Professional physical activity logger to a discrete accelerometer-based activity monitor. Medical Engineering \& Physics, 29, 930-934. doi:10.1016/i.medengphy.2006.10.001

[8] Ryan, C.G., Grant, P.M., Tigbe, W.W. and Granat, M.H. (2006) The validity and reliability of a novel activity monitor as a measure of walking. British Journal of Sports Medicine, 40, 779-784. doi:10.1136/bjsm.2006.027276

[9] Maddocks, M., Petrou, A., Skipper, L. and Wilcock, A. (2008) Validity of three accelerometers during treadmill walking and motor vehicle travel. British Journal of Sports Medicine, Online First, doi:10.1136/bjsm.2008.051128
[10]Oliver, M., Badland, H.M., Schofield, G.M. and Shepherd, J. (in press) Identification of non-wear time and sedentary behavior using accelerometry. Research Quarterly for Exercise and Sport.

[11]Oliver, M., Schofield, G.M., Badland, H.M. and Shepherd, J. (2010) Utility of accelerometer thresholds for classifying sitting in office workers. Preventive Medicine, 51, 357360. doi:10.1016/j.ypmed.2010.08.010

[12] Crouter, S.E., Schneider, P.L., Karabulut, M. and Bassett, D.R., Jr. (2003) Validity of 10 electronic pedometers for measuring steps, distance, and energy cost. Medicine \& Science in Sports \& Exercise, 35, 1455-1460. doi:10.1249/01.MSS.0000078932.61440.A2

[13] Le Masurier, G.C., Lee, S.M. and Tudor-Locke, C. (2004) Motion sensor accuracy under controlled and free-living conditions. Medicine \& Science in Sports \& Exercise, 36, 905-910. doi:10.1249/01.MSS.0000126777.50188.73

[14] Bland, J.M. and Altman, D.G. (1999) Measuring agreement in method comparison studies. Statistical Methods in Medical Research, 8, 135-160. doi:10.1191/096228099673819272

[15] Bland, J.M. and Altman, D.G. (2003) Applying the right statistics: Analyses of measurement studies. Ultrasound in Obstetrics and Gynecology, 22, 85-93. doi:10.1002/uog.122

[16] Brown, M.B. and Forsythe, A.B. (1974) Robust tests for equality of variances. Journal of the American Statistical Association, 69, 364-367. doi:10.2307/2285659

[17] Tudor-Locke, C., Giles-Corti, B., Knuiman, M. and McCormack, G. (2008) Tracking of pedometer-determined physical activity in adults who relocate: Results from RESIDE. International Journal of Behavioral Nutrition and Physical Activity, 5, doi:10.1186/1479-5868-5-39

[18] Tudor-Locke, C.E. and Myers, A.M. (2001) Methodological considerations for researchers and practitioners using pedometers to measure physical (ambulatory) activity. Research Quarterly for Exercise and Sport, 72, 1-12.

[19] Tudor-Locke, C., Myers, A.M., Bell, R.C., Harris, S.B. and Rodger, N.W. (2002) Preliminary outcome evaluation of the First Step Program: a daily physical activity intervention for individuals with type 2 diabetes. Patient Education and Counseling, 47, 23-28. doi:10.1016/S0738-3991(01)00169-0

[20] Tudor-Locke, C. and Bassett Jr, D.R. (2004) How many steps/ day are enough? Preliminary pedometer indices for public health. Sports Medicine, 34, 1-8. doi:10.2165/00007256-200434010-00001

[21] Crouter, S.E., Schneider, P.L. and Bassett, D.R. (2005) Spring-Levered versus Piezo-Electric Pedometer Accuracy in Overweight and Obese Adults. Medicine \& Science in Sports \& Exercise, 37, 1673-1679. doi:10.1249/01.mss.0000181677.36658.a8

[22] Levine, J.A., McCrady, S.K., Lanningham-Foster, L.M., Kane, P.H., Foster, R.C. and Manohar, C.U. (2008) The role of free-living daily walking in human weight gain and obesity. Diabetes, 57, 548-554. doi:10.2337/db07-0815 